\title{
Impact of Major Pulmonary Resections on Right Ventricular Function: Early Postoperative Changes
}

\author{
Hany M. Elrakhawy, MD, ${ }^{1,2}$ Mohamed A. Alassal, MD, ${ }^{1,3}$ Ayman M. Shaalan, MD, ${ }^{1,4}$ \\ Ahmed A. Awad, MB.BCh, ${ }^{5}$ Sameh Sayed, MD, ${ }^{6}$ Mohammad M. Saffan, MD ${ }^{1}$ \\ ${ }^{1}$ Cardiothoracic Surgery Department, Benha University, Benha, Egypt; ${ }^{2}$ Prince Mutaib Bin Abdul-Aziz Hospital, Sakaka, Al-Jouf, \\ Saudi Arabia; ${ }^{3}$ King Fahd Medical City, Riyadh, Saudi Arabia; ${ }^{4}$ Dallah Hospital, Cardiac Center, Riyadh, Saudi Arabia; ${ }^{5}$ Dallah \\ Hospital, Endocrinology Center, Riyadh, Saudi Arabia; ${ }^{6}$ Cardiothoracic Surgery Department, Assiut University, Assiut, Egypt
}

\section{ABSTRACT}

Background: Right ventricular (RV) dysfunction after pulmonary resection in the early postoperative period is documented by reduced RV ejection fraction and increased RV end-diastolic volume index. Supraventricular arrhythmia, particularly atrial fibrillation, is common after pulmonary resection. RV assessment can be done by non-invasive methods and/ or invasive approaches such as right cardiac catheterization. Incorporation of a rapid response thermistor to pulmonary artery catheter permits continuous measurements of cardiac output, right ventricular ejection fraction, and right ventricular end-diastolic volume. It can also be used for right atrial and right ventricular pacing, and for measuring right-sided pressures, including pulmonary capillary wedge pressure.

Methods: This study included 178 patients who underwent major pulmonary resections, 36 who underwent pneumonectomy assigned as group (I) and 142 who underwent lobectomy assigned as group (II). The study was conducted at the cardiothoracic surgery department of Benha University hospital in Egypt; patients enrolled were operated on from February 2012 to February 2016. A rapid response thermistor pulmonary artery catheter was inserted via the right internal jugular vein. Preoperatively the following was recorded: central venous pressure, mean pulmonary artery pressure, pulmonary capillary wedge pressure, cardiac output, right ventricular ejection fraction and volumes. The same parameters were collected in fixed time intervals after 3 hours, 6 hours, 12 hours, 24 hours, and 48 hours postoperatively.

Results: For group (I): There were no statistically significant changes between the preoperative and postoperative records in the central venous pressure and mean arterial pressure; there were no statistically significant changes in the preoperative and 12, 24, and 48 hour postoperative records for cardiac index; 3 and 6 hours postoperative showed significant changes. There were statistically significant changes between the preoperative and postoperative records for heart rate,

Received Fune 30, 2017; received in revised form August 16, 2017; accepted August 24, 2017.

Correspondence: Hany Mohamed Elrakhawy, MD, Assistant Professor of Cardiothoracic Surgery, Benha Faculty of Medicine, Benha University, Egypt o Consultant of Thoracic Surgery, Prince Mutaib Bin Abdul-Aziz Hospital, Sakaka, Al-7ouf, Saudi Arabia; (e-mail: hanyelrakbawy@yaboo.com). mean pulmonary artery pressure, pulmonary capillary wedge pressure, pulmonary vascular resistance, right ventricular ejection fraction and right ventricular end diastolic volume index, in all postoperative records. For group (II): There were no statistically significant changes between the preoperative and all postoperative records for the central venous pressure, mean arterial pressure and cardiac index. There were statistically significant changes between the preoperative and postoperative records for heart rate, mean pulmonary artery pressure, pulmonary capillary wedge pressure, pulmonary vascular resistance, right ventricular ejection fraction and right ventricular end diastolic volume index in all postoperative records. There were statistically significant changes between the two groups in all postoperative records for heart rate, mean pulmonary artery pressure, pulmonary capillary wedge pressure, pulmonary vascular resistance, right ventricular ejection fraction and right ventricular end diastolic volume index.

Conclusion: There is right ventricular dysfunction early after major pulmonary resection caused by increased right ventricular afterload. This dysfunction is more present in pneumonectomy than in lobectomy. Heart rate, mean pulmonary artery pressure, pulmonary capillary wedge pressure, pulmonary vascular resistance, right ventricular ejection

Table 1. Demographic Data and Etiologies

\begin{tabular}{lccc}
\hline & $\begin{array}{c}\text { Pneumonectomy } \\
(\mathrm{n}=36)\end{array}$ & $\begin{array}{c}\text { Lobectomy } \\
(\mathrm{n}=142)\end{array}$ & $P$ \\
\hline Age, y & $66.3 \pm 7.4$ & $62.9 \pm 9.7$ & .051 \\
Sex (male) & $26(72.2)$ & $97(68.3)$ & NA \\
BMI & $29.5 \pm 6.3$ & $31.5 \pm 1.6$ & .057 \\
Smoking & $30(83.3)$ & $95(66.9)$ & NA \\
Diabetes mellitus & $21(58.3)$ & $45(31.7)$ & NA \\
Bronchogenic carcinoma & $27(75)$ & $83(58.5)$ & NA \\
Lobar emphysema & $0(0.0)$ & $16(11.3)$ & NA \\
Traumatic & $2(5.6)$ & $7(4.9)$ & NA \\
Pulmonary infection & $7(19.4)$ & $36(25.4)$ & NA \\
\hline
\end{tabular}

Data is presented as mean $\pm \mathrm{SD}$ or $\mathrm{n}(\%)$. 
Table 2. Pneumonectomy $(n=36)$

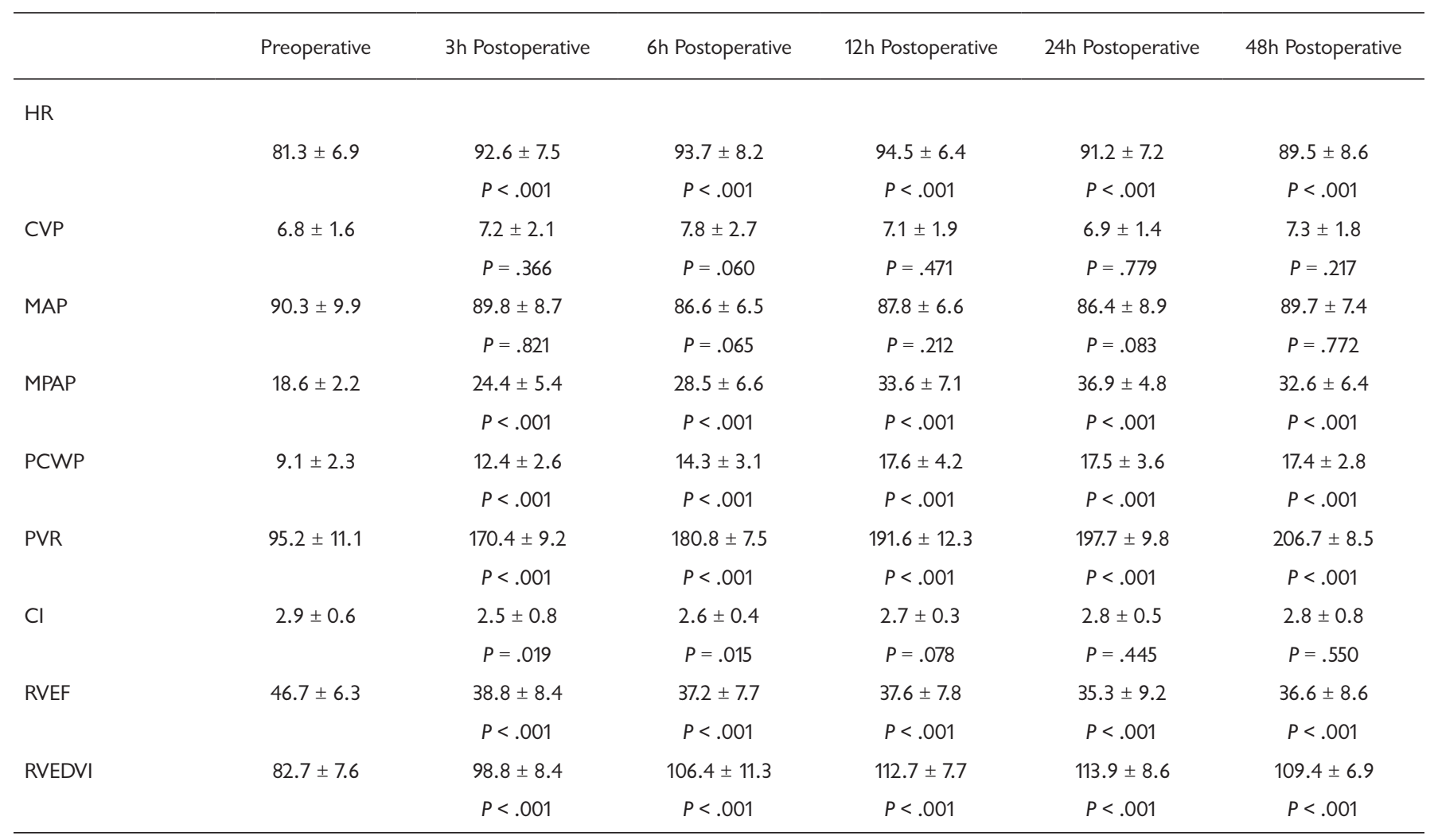

HR, heart rate; CVP, central venous pressure; MAP, mean arterial pressure; MPAP, mean pulmonary artery pressure; Cl, cardiac index; PCWP, pulmonary capillary wedge pressure; PVR, peripheral vascular resistance; RVEF, right ventricular ejection fraction; RVEDVI, right ventricular end diastolic volume index; $\mathrm{P}$, $\mathrm{p}$ value: it's significant if $p<0.05$

fraction, and right ventricular end diastolic volume index are significantly affected by pulmonary resection.

\section{INTRODUCTION}

Right ventricular (RV) dysfunction after pulmonary resection in the early postoperative period occurs is documented by reduced RV ejection fraction (RVEF) and increased RV end-diastolic volume index (RVEDVI). It's speculated that this RV dysfunction may be due to alterations in RV contractility or changes in RV afterload, predisposing patients to postoperative complications and long-term morbidity [McCall 2016, Reed 1996].

Since supraventricular tachyarrhythmia (SVT), particularly atrial fibrillation (AF), is common after pulmonary resection, it's suggested that marked RV distention is a contributing mechanism to development of SVT. Risk factors for the development of SVT after noncardiac thoracic surgery include increasing age; male sex; previous history of cardiac disease; history of hypertension; malignant disease; anesthetic agents; extent of pulmonary resection; mediastinal lymph node dissection; intrapericardial pneumonectomy; intraoperative cardiac arrest; intraoperative blood transfusions; electrolyte imbalance; pulmonary complications and need for repeat thoracotomy. This arrhythmia is usually treatable and does not affect postoperative morbidity and mortality significantly [Elrakhawy 2014, Kowalewski 1999].

The RV can be assessed by non-invasive methods as echocardiography-Doppler, isotopic technology, cardiac magnetic resonance imaging and/or invasive approaches as right cardiac catheterization (RHC) [Fayssoil 2009].

RHC was applied for the first time by Dr. Werner Forssmann. In 1929, he introduced a catheter into the right atrium of his own heart to establish the feasibility of right heart catheterization in humans. Catheters that could be advanced into the pulmonary arteries were developed by Cournand and Richards. Forssmann, Cournand, and Richards received the Nobel Prize in medicine for their discoveries in 1956. The balloon flotation catheters were invented by Drs. Swan and Ganz in 1970, popularly known as "Swan-Ganz" catheters. The conventional pulmonary artery catheter (PAC) was developed by incorporation of a rapid response thermistor that permitted continuous measurements of cardiac output (CO), right ventricular ejection fraction (RVEF), and right ventricular end-diastolic volume (RVEDV). It is also used for right atrial and right ventricular pacing, and for measuring right-sided pressures, including pulmonary capillary wedge pressure (PCWP) [Rosenkranz 2015; Chatterjee 2009; Robin 2006; Marik 2013]. 
Table 3. Lobectomy $(n=142)$

\begin{tabular}{|c|c|c|c|c|c|c|}
\hline \multicolumn{7}{|l|}{$H R$} \\
\hline & & $P<.001$ & $P<.001$ & $P<.001$ & $P<.001$ & $P=.001$ \\
\hline CVP & $7.3 \pm 2.8$ & $7.4 \pm 2.6$ & $7.5 \pm 2.3$ & $7.3 \pm 1.9$ & $7.2 \pm 2.1$ & $7.5 \pm 2.6$ \\
\hline \multirow[t]{2}{*}{ MAP } & $87.2 \pm 8.8$ & $86.8 \pm 9.3$ & $89.3 \pm 9.4$ & $88.6 \pm 6.8$ & $86.4 \pm 7.1$ & $87.7 \pm 6.4$ \\
\hline & & $P=.710$ & $P=.053$ & $P=.135$ & $P=.400$ & $P=.584$ \\
\hline \multirow[t]{2}{*}{ MPAP } & $16.9 \pm 5.3$ & $19.8 \pm 5.6$ & $22.3 \pm 4.6$ & $23.8 \pm 8.1$ & $25.6 \pm 6.8$ & $24.9 \pm 7.3$ \\
\hline & & $P<.001$ & $P<.001$ & $P<.001$ & $P<.001$ & $P<.001$ \\
\hline PCWP & $8.6 \pm 2.4$ & $9.4 \pm 3.1$ & $9.8 \pm 2.8$ & $10.3 \pm 4.2$ & $9.7 \pm 3.4$ & $9.6 \pm 3.3$ \\
\hline \multirow[t]{2}{*}{$\mathrm{Cl}$} & $3.2 \pm 0.9$ & $3.0 \pm 0.9$ & $3.1 \pm 0.3$ & $3.1 \pm 0.5$ & $3.1 \pm 0.7$ & $3.1 \pm 0.2$ \\
\hline & & $P=.062$ & $P=.210$ & $P=.248$ & $P=.297$ & $P=.197$ \\
\hline \multirow[t]{2}{*}{ RVEF } & $49.6 \pm 9.8$ & $44.2 \pm 9.1$ & $43.8 \pm 8.5$ & $43.3 \pm 9.2$ & $41.9 \pm 8.7$ & $42.3 \pm 7.6$ \\
\hline & & $P<.001$ & $P<.001$ & $P<.001$ & $P<.001$ & $P<.001$ \\
\hline \multirow[t]{2}{*}{ RVEDVI } & $79.8 \pm 8.4$ & $91.7 \pm 5.8$ & $96.3 \pm 6.4$ & $99.2 \pm 7.1$ & $94.7 \pm 6.5$ & $95.5 \pm 8.2$ \\
\hline & & $P<.001$ & $P<.001$ & $P<.001$ & $P<.001$ & $P<.001$ \\
\hline
\end{tabular}

HR, heart rate; CVP, central venous pressure; MAP, mean arterial pressure; MPAP, mean pulmonary artery pressure; Cl, cardiac index; PCWP, pulmonary capillary wedge pressure; PVR, peripheral vascular resistance; RVEF, right ventricular ejection fraction; RVEDVI, right ventricular end diastolic volume index; $\mathrm{P}$, $\mathrm{p}$ value: it's significant if $p<0.05$

The aim of this study was to evaluate and to compare the effect of major pulmonary resection (lobectomy and pneumonectomy) on right ventricular function with a comparison between the effects of these resections in patients undergoing either lobectomy or pneumonectomy.

\section{PATIENT AND METHODS}

This is a retrospective study. All data were collected after getting the approval of the ethical committee. A written consent from patients to utilize their data and images in any publication for scientific purpose is a part of the patients' admission file, and it was signed by all patients enrolled in this work.

After review of these patients' files, 178 patients underwent major pulmonary resection; 36 underwent pneumonectomy assigned as group (I) and 142 underwent lobectomy assigned as group (II). Patients selected were required to have normal values of the studied parameters preoperatively, with no statistical significant difference between the two groups for the preoperative records. The study was conducted at the cardiothoracic surgery department of Benha University hospital in Egypt. Patients enrolled were operated on from February 2012 to February 2016.

After induction of anesthesia, all patients were intubated by left sided double lumen endotracheal tube; and the radial artery was cannulated for continuous recording of arterial blood pressure and arterial blood gases. Using Seldinger technique, a sheath was inserted over guide wire and dilator into the right internal jugular vein, the guide wire and dilator then removed. After testing the balloon of a rapid response thermistor, multi-lumen pulmonary artery catheter $(7.5 \mathrm{~F} \times$ $110 \mathrm{~cm}$ ) was advanced through the sheath.

Before starting the planned operation, preoperative records were taken, and postoperative data were collected in fixed time intervals after 3 hours, 6 hours, 12 hours, 24 hours, and 48 hours. Exclusion criteria included all cases with: (idiopathic or secondary) pulmonary hypertension or cor pulmonale, with impaired RV (right ventricular) function, chronic arrhythmias, history of arrhythmia within one month preoperatively, history of myocardial infarction or chronic heart disease either acquired or congenital and patients who underwent VATS (video-assisted thoracoscopic surgery) for lung resection.

\section{Statistical Analysis}

SPSS version 22.0 was used for data analysis. Continuous variables were expressed as the mean \pm standard deviation (SD), depending on the distribution of the data; either the unpaired t-test or the Mann-Whitney $\mathrm{U}$ test was used for the 
Table 4. Pneumonectomy Versus Lobectomy

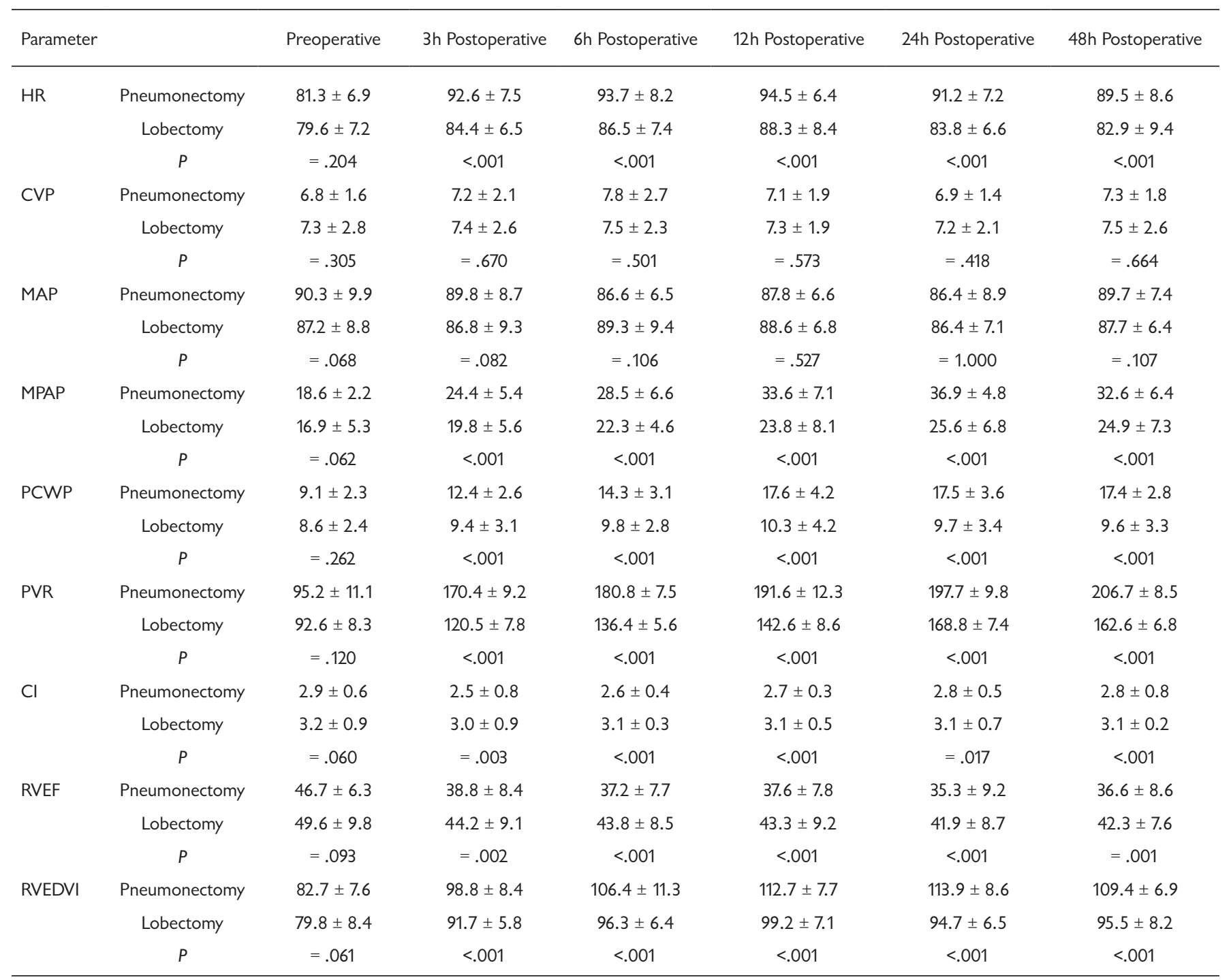

HR, heart rate; CVP, central venous pressure; MAP, mean arterial pressure; MPAP, mean pulmonary artery pressure; Cl, cardiac index; PCWP, pulmonary capillary wedge pressure; PVR, peripheral vascular resistance; RVEF, right ventricular ejection fraction; RVEDVI, right ventricular end diastolic volume index; $\mathrm{P}$, $\mathrm{P}$ value: it's significant if $p<0.05$

intergroup comparisons. Categorical data were expressed as the number and (percentage), and were compared using the Pearson chi-square and Fisher exact tests. Repeated measures analysis of variance (ANOVA) was used to compare hemodynamic and cardiac measurement variables through baseline and postoperative 5 time-frames. All reported $\mathrm{P}$ values were two-sided, and a value of $P<.05$ was considered statistically significant.

\section{RESULTS}

This is a retrospective study, which included 178 patients who underwent pulmonary resection. Patients were divided into two groups: group (I) included $36(20.2 \%)$ patients who underwent pneumonectomy; group (II) included 142
(79.8\%) patients who underwent anatomical pulmonary lobectomy. Patients' demographic data and etiologies are shown in Table 1.

Heart rate (HR), central venous pressure (CVP), mean arterial pressure (MAP), mean pulmonary artery pressure (MPAP), pulmonary capillary wedge pressure (PCWP), pulmonary vascular resistance (PVR), cardiac index (CI), right ventricular ejection fraction (RVEF) and right ventricular end-diastolic volume index (RVEDVI) were taken as parameters for assessment of right ventricular function. Preoperative records of these parameters were taken and were compared to postoperative records taken after 3, 6, 12,24 , and 48 hours.

For group (I): There were no statistically significant changes between the preoperative and postoperative records 
Table 5. Hemodynamic Intergroup Comparison

\begin{tabular}{|c|c|c|c|c|c|c|c|c|}
\hline HR & Lobectomy & $79.6 \pm 7.2$ & $84.4 \pm 6.5$ & $86.5 \pm 7.4$ & $88.3 \pm 8.4$ & $83.8 \pm 6.6$ & $82.9 \pm 9.4$ & \\
\hline CVP & Pneumonectomy & $6.8 \pm 1.6$ & $7.2 \pm 2.1$ & $7.8 \pm 2.7$ & $7.1 \pm 1.9$ & $6.9 \pm 1.4$ & $7.3 \pm 1.8$ & .768 \\
\hline MAP & Lobectomy & $87.2 \pm 8.8$ & $86.8 \pm 9.3$ & $89.3 \pm 9.4$ & $88.6 \pm 6.8$ & $86.4 \pm 7.1$ & $87.7 \pm 6.4$ & \\
\hline \multirow[t]{2}{*}{ MPAP } & Pneumonectomy & $18.6 \pm 2.2$ & $24.4 \pm 5.4$ & $28.5 \pm 6.6$ & $33.6 \pm 7.1$ & $36.9 \pm 4.8$ & $32.6 \pm 6.4$ & $<.001$ \\
\hline & Lobectomy & $16.9 \pm 5.3$ & $19.8 \pm 5.6$ & $22.3 \pm 4.6$ & $23.8 \pm 8.1$ & $25.6 \pm 6.8$ & $24.9 \pm 7.3$ & \\
\hline PCWP & Pneumonectomy & $9.1 \pm 2.3$ & $12.4 \pm 2.6$ & $14.3 \pm 3.1$ & $17.6 \pm 4.2$ & $17.5 \pm 3.6$ & $17.4 \pm 2.8$ & $<.001$ \\
\hline \multirow[t]{2}{*}{$\mathrm{Cl}$} & Pneumonectomy & $2.9 \pm 0.6$ & $2.5 \pm 0.8$ & $2.6 \pm 0.4$ & $2.7 \pm 0.3$ & $2.8 \pm 0.5$ & $2.8 \pm 0.8$ & .013 \\
\hline & Lobectomy & $3.2 \pm 0.9$ & $3.0 \pm 0.9$ & $3.1 \pm 0.3$ & $3.1 \pm 0.5$ & $3.1 \pm 0.7$ & $3.1 \pm 0.2$ & \\
\hline \multirow[t]{2}{*}{ RVEF } & Pneumonectomy & $46.7 \pm 6.3$ & $38.8 \pm 8.4$ & $37.2 \pm 7.7$ & $37.6 \pm 7.8$ & $35.3 \pm 9.2$ & $36.6 \pm 8.6$ & $<.001$ \\
\hline & Lobectomy & $49.6 \pm 9.8$ & $44.2 \pm 9.1$ & $43.8 \pm 8.5$ & $43.3 \pm 9.2$ & $41.9 \pm 8.7$ & $42.3 \pm 7.6$ & \\
\hline \multirow[t]{2}{*}{ RVEDVI } & Pneumonectomy & $82.7 \pm 7.6$ & $98.8 \pm 8.4$ & $106.4 \pm 11.3$ & $112.7 \pm 7.7$ & $113.9 \pm 8.6$ & $109.4 \pm 6.9$ & $<.001$ \\
\hline & Lobectomy & $79.8 \pm 8.4$ & $91.7 \pm 5.8$ & $96.3 \pm 6.4$ & $99.2 \pm 7.1$ & $94.7 \pm 6.5$ & $95.5 \pm 8.2$ & \\
\hline
\end{tabular}

$\mathrm{HR}$, heart rate; CVP, central venous pressure; MAP, mean arterial pressure; MPAP, mean pulmonary artery pressure; Cl, cardiac index; PCWP, pulmonary capillary wedge pressure; PVR, peripheral vascular resistance; RVEF, right ventricular ejection fraction; RVEDVI, right ventricular end diastolic volume index; P, pvalue: it's significant if $p<0.05$

in the CVP and MAP. There were no statistically significant changes in the preoperative and 12,24 , and 48 hour postoperative records of CI, while 3 and 6 hour postoperative measurements showed significant changes. There were statistically significant changes between the preoperative and postoperative records of HR, MPAP, PCWP, PVR, RVEF and RVEDVI in all postoperative records as shown in Table 2.

For group (II): There were no statistically significant changes between the preoperative and all postoperative records in CVP, MAP, and CI. There were statistically significant changes between the preoperative and postoperative records of HR, MPAP, PCWP, PVR, RVEF and RVEDVI in all postoperative records as shown in Table 3 .

By comparing the results of the two groups, there were no statistically significant changes between the preoperative records for all parameters. There were no statistically significant changes between all postoperative records in the CVP and MAP. There were statistically significant changes between all postoperative records for HR, MPAP, CI, PCWP, PVR, RVEF, and RVEDVI in all postoperative records as shown in Tables 4 and 5.

Figures 1-9 illustrate the intergroup differences between group (I) and group (II) in the preoperative and all postoperative hours' recordings for HR, CVP, MAP, MPAP, CI, PCWP, PVR, RVEF, and RVEDVI.

\section{DISCUSSION}

Lung resections are common thoracic surgical procedures that can be done at any age for variable etiologies. Various extensions of resection can be done, including pneumonectomy, lobectomy, or segmentectomy. Many studies have been done to assess the impact of these resections on the focused hemodynamics. We focused our study on documenting these hemodynamic changes in early stages after resection. Also, we designed our study to differentiate between pneumonectomy and lobectomy RV function through analysis of records taken by pulmonary artery catheter.

The association between cardiac arrhythmias and pulmonary resection, especially above age 45 years, has been well known for many decades. Rhythm disturbance can occur intra- or postoperative, and may be in the form of multiple extra-systoles, atrial flutter, or atrial fibrillation which is the most common rhythm disturbance [Hurt 1958, Kim 2006, Sato 2014].

In our study, there was a statistically significant increase in the heart rate when comparing the pre- and postoperative records of both groups. Also, there was a statistically significant increase in the heart rate in the pneumonectomy group in comparison with the lobectomy group postoperatively. SVT was recorded in $22(15.5 \%)$ patients who underwent lobectomy and in $12(33.3 \%)$ patients who underwent pneumonectomy. These results are very close to that reported by 


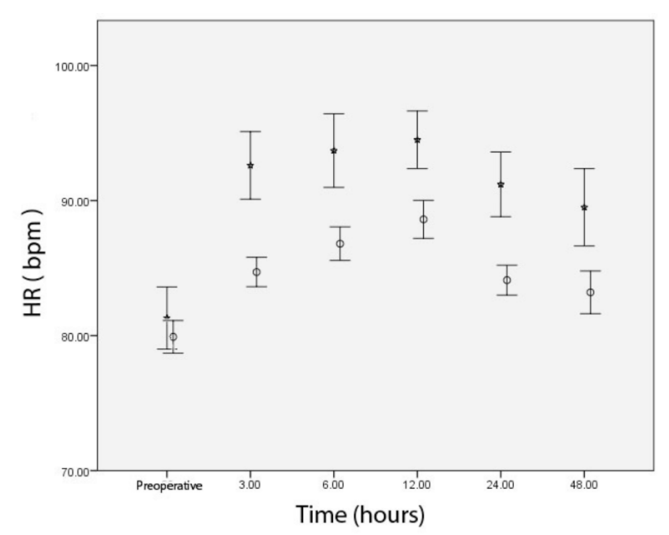

Figure 1. Heart rate mean and $95 \%$ confidence interval for both groups preoperatively and postoperatively. HR indicates heart rate; bpm: beat per minute. + indicates pneumonectomy group; \$ lobectomy group.

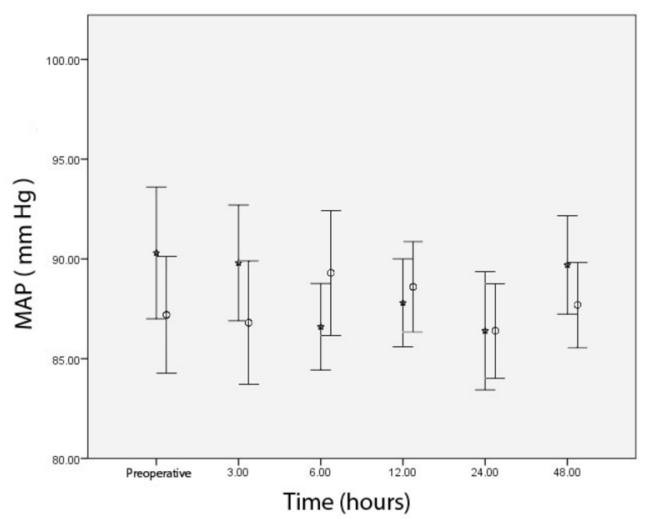

Figure 3. MAP mean and $95 \%$ confidence interval for both groups preoperatively and postoperatively. MAP indicates mean arterial pressure. $\downarrow$ indicates pneumonectomy group; $\phi$ lobectomy group.

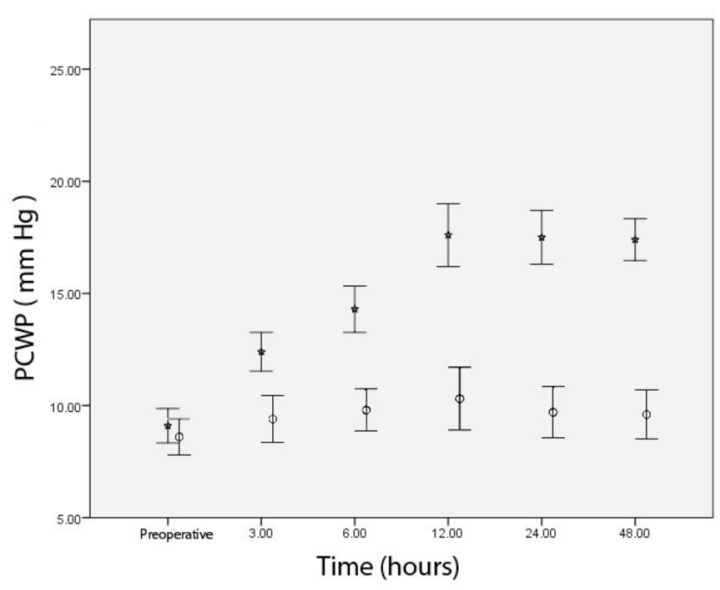

Figure 5. PCWP mean and $95 \%$ confidence interval for both groups preoperatively and post operatively. PCWP indicates pulmonary capillary wedge pressure. $\downarrow$ indicates pneumonectomy group; ф lobectomy group.

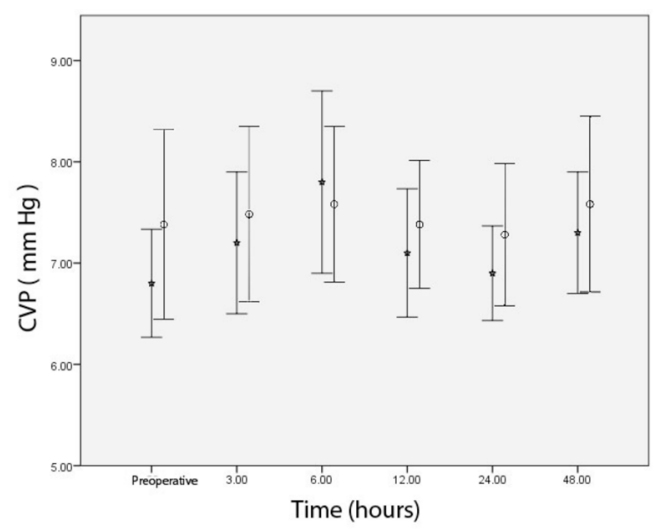

Figure 2. CVP mean and $95 \%$ confidence interval for both groups preoperatively and postoperatively. CVP indicates central venous pressure. ; indicates pneumonectomy group; \$ lobectomy group.

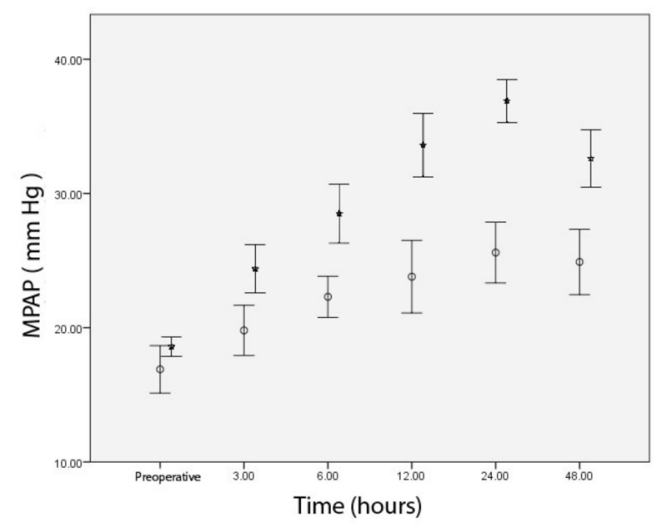

Figure 4. MPAP mean and $95 \%$ confidence interval for both groups preoperatively and postoperatively. MPAP indicates mean pulmonary arterial pressure. $\downarrow$ indicates pneumonectomy group; $\uparrow$ lobectomy group.

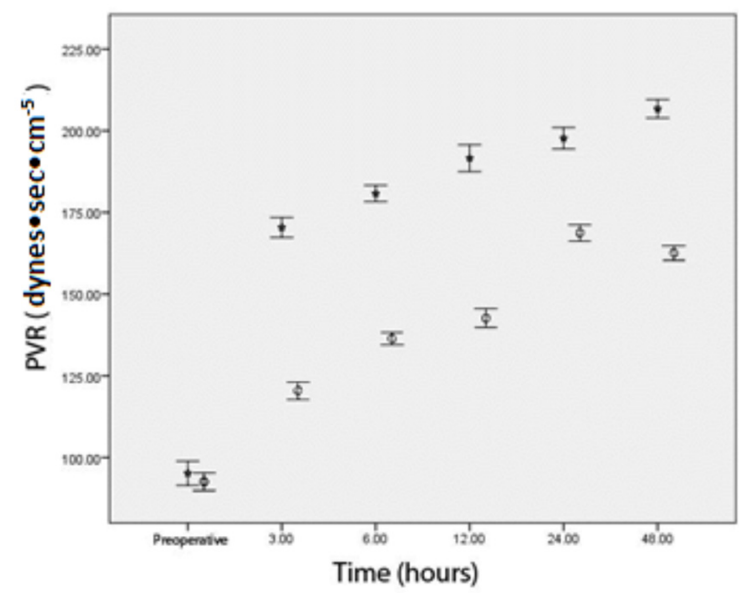

Figure 6. PVR mean and $95 \%$ confidence interval for both groups preoperatively and postoperatively. PVR indicates pulmonary vascular resistance. + indicates pneumonectomy group; \& lobectomy group. 


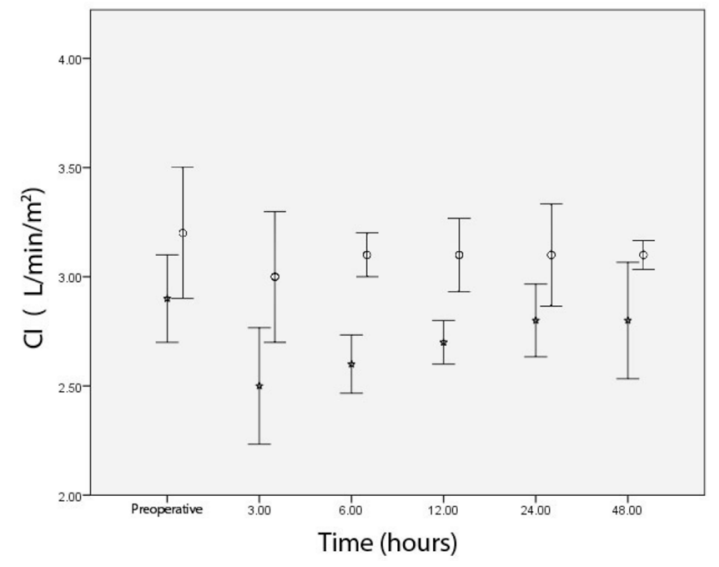

Figure 7. Cardiac index mean and $95 \%$ confidence interval for both groups preoperatively and postoperatively. $\mathrm{Cl}$ indicates cardiac index. † indicates pneumonectomy group; $\$$ lobectomy group.

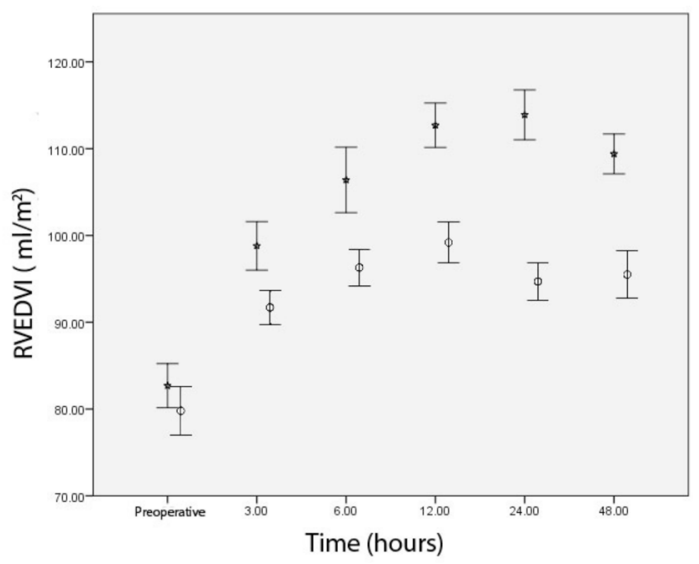

Figure 9. RVEDVI mean and $95 \%$ confidence interval for both groups preoperatively and postoperatively. RVEDVI indicates right ventricle end-diastolic volume index. + indicates pneumonectomy group;

ф lobectomy group.

the same author in another study, as the reported SVT was $14.8 \%$ after lobectomy, and $30.5 \%$ after pneumonectomy [Elrakhawy 2014]. There was an intraoperative episode of $\mathrm{AF}$ in $2(5.6 \%)$ patients who underwent pneumonectomy and in $5(3.5 \%)$ patients who underwent lobectomy; these results are also close to that reported in another study in which the reported intraoperative $\mathrm{AF}$ was $3.52 \%$ and $4.8 \%$ for lobectomy and pneumonectomy [ $\mathrm{Wu} 2012]$. In our study, the postoperative records showed development of AF in 15 (10.6\%) patients who underwent lobectomy and in $8(22.2 \%)$ patients who underwent pneumonectomy. These results are less than that reported by other authors, who reported $18 \%$ and $23 \%$ after lobectomy and $30 \%$ and $35 \%$ after pneumonectomy [Vaporciyan2004; Rena 2001].

Comparing pre- and postoperative records of MPAP, there was a significant increase in the postoperative readings in both groups. There was also a significant change when comparing the postoperative results of the two groups. In spite of these

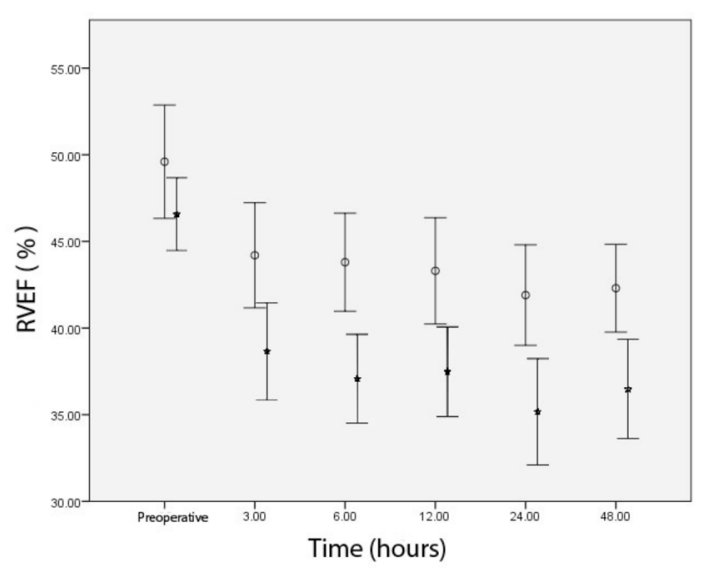

Figure 8. RVEF mean and $95 \%$ confidence interval for both groups preoperatively and postoperatively. RVEF indicates right ventricle ejection fraction. . indicates pneumonectomy group; \& lobectomy group.

significant changes, there was no pulmonary hypertension in the lobectomy group, with a mild form of pulmonary hypertension in the 12,24 , and 48 postoperative hour readings in the pneumonectomy group, with pulmonary hypertension defined as MPAP > $30 \mathrm{mmHg}$ [Wei 2014]. Other authors concluded that lobectomy and pneumonectomy increased the pulmonary artery pressure but without statistical significance [Behzadnia 2004; Cumbo-Nacheli 2013].

Pulmonary edema (PE) following pulmonary resection is a rare but serious complication with mortality of more than $50 \%$. It is more frequent post-pneumonectomy than post lobectomy, and the term post-pneumonectomy pulmonary edema was first described in 1984 by Zeldin et al. [Zeldin 1984, Slinger 1999]. Thus, intravenous (IV) fluid management is of significant importance for all patients scheduled for pulmonary resection; management guided by CVP should be considered to avoid pulmonary edema from over hydration in the perioperative period and this should be done on an individual basis [Chau 2014; Tsuchida 2001; Kutlu 2000].

In our study, there was a wide range of variability in the fluid management of patients. Regarding CVP and MAP there were no significant change in the preoperative and all postoperative records for the pneumonectomy and lobectomy groups. There was no significant change in all postoperative records between the two groups. This may be due to the variability of the fluid management for patients, which was individualized for each case. Also, there was no early postoperative pulmonary edema in the lobectomy group, with mild to moderate in 4 cases $(11.1 \%)$ in the pneumonectomy group that occurred after 6 hours in 3 patients and after 24 hours in 1 patient, with no difference on the side of pneumonectomy (2 underwent right and 2 underwent left pneumonectomy). The incidence of post-pneumonectomy pulmonary edema in our series was less than reported in another study in which $15 \%$ of patients developed PE [Parquin 1996]; but it was higher than that reported in a study in which pulmonary edema occurred in $5.1 \%$ of right pneumonectomies, and in $4.0 \%$ of left pneumonectomies. The same study reported pulmonary edema in 
$1 \%$ of lobectomies [Waller 1993], while in our study no cases developed pulmonary edema after lobectomy.

Regarding PCWP and PVR patients, group (I) showed significant increase postoperatively, in comparison to the preoperative data in the same group. Also, the readings of these parameters were increased post-lung resection in group (II). The degree of this increase was higher in group (I) than group (II) with significant difference in between after ligation of the vascular pedicle, leading to reduction of vascular bed for blood flow from the right ventricle. These measurements were taken by Swan-Ganz catheter through analysis of data after wedging the balloon within the pulmonary artery, and this reflected the increase in the afterload against the right ventricle which had small muscle mass; one of the studies proved that, at the time of pulmonary artery clamping, right ventricular ejection fraction of less than $35 \%$, pulmonary vascular resistance greater than or equal to 200 dyne.sec.cm-5, and a pulmonary vascular resistance/right ventricular ejection fraction ratio greater than or equal to 5.0 predicted the development of long-term cardiopulmonary disability[Lewis 1994].

Others suggested that a change in afterload may be the main determinant of the deterioration in right ventricular pump performance after major pulmonary resection[Okada 1994]. In our study, CI showed minimal change in both groups of patients, especially in the pneumonectomy group, which was reduced until the first 6 hours before returning to its previous range; while in lobectomy cases, CI was reduced in the first reading after 3 hours, and then physiological adaptation allowed the return to previous baseline values later. Other authors found that in the acute post-resection period (up to 2 hours postoperatively) there is right and left ventricular dysfunction [Mageed 2005].

Analysis of patient data for RVEF and RVEDVI revealed that there was significant reduction of RVEF over all time frames in both groups, which were more marked in pneumonectomy cases than in lobectomy cases by thermodilution method. This is mostly explained by the increase in PCWP and PVR (increase in the afterload). This was compensated in group II more than group I as there was a large surface area of resistance resected; also, there was some circulatory volume blood loss. One of the studies noted that elevation of the right ventricular afterload associated with a decrease of pulmonary vascular bed is a serious problem immediately after pneumonectomy, which is compensated by increasing right ventricular work and decreasing circulating blood volume[Uno 1993]. This was reflected in the increase of RVEDVI in both groups significantly, and was higher also in pneumonectomy cases versus lobectomy cases. In an analysis comparing lobectomy patients and pneumonectomy cases others found a result of a more pronounced and sustained deterioration in right ventricular hemodynamics of RVEDVI and RVEF [Boldt 1996]. This is because of dilatation and distension of the thin RV and some degree of impaired blood supply to the distended ventricle. This is why diuretics play an important role during management of cases after lung resection, especially in cases of pneumonectomy. There is definitely a degree of RV dysfunction post-lung resection with either pneumonectomy or lobectomy cases. Therefore, surgeons must consider that before any procedure, so RV assessment is an important indicator for outcome of patients after lung resection, and these evaluations can be performed by TTE.

\section{Conclusion}

Our study demonstrates that there is right ventricular dysfunction early after major pulmonary resection as evidenced by increased RV afterload. This dysfunction is more pronounced in pneumonectomy than in lobectomy. HR, MPAP, PCWP, PVR, RVEF, and RVEDVI are significantly affected by pulmonary resection, while CVP, MAP, and CI are minimally affected. It is recommended that right ventricular function be assessed preoperatively by echocardiography.

\section{REFERENCES}

Behzadnia N, Sharif-Kashani B, Javaherzadeh M. 2004. Effect of major lung resection on pulmonary artery pressure. Tanaffos 3:23-27.

Boldt J ,Muller M ,Uphus D, Padberg W, Hempelmann G. 1996. Cardiorespiratory changes in patients undergoing pulmonary resection using different anesthetic management techniques. J Cardiothoracic Vasc Anesth 10(7):854-9.

Chatterjee K. 2009. The Swan-Ganz catheters: past, present, and future. A viewpoint. Circulation 119:147-52.

Chau EH, Slinger P. 2014. Perioperative fluid management for pulmonary resection surgery and esophagectomy. Semin Cardiothorac Vasc Anesth 18:36-44.

Cumbo-Nacheli G, Tonelli A, Dweik RA. 2013. Effect of pneumonectomy on pulmonary artery pressure and right ventricular function. Chirurgia 26:73-8.

Elrakhawy HM, Alassa MA, Elsadeck N, Shaalan A, Ezeldin TH, Shalabi A. 2014. Predictive factors of supraventricular arrhythmias after noncardiac thoracic surgery: a multicenter study. Heart Surg Forum 17:E308-12.

Fayssoil A, Abasse S, Nardi O. 2009. Right ventricular assessment with echocardiography. Med Sci (Paris). 25:513-8. [Article in French]

Hurt RL, Bates M. 1958. The value of quinidine in the prevention of cardiac arrhythmias after pulmonary resection. Thorax 13(1):39-41.

Kim HJ, Lee SK, Kim YM, Moon HS. 2006. Pharmacological Cardioversion with Phenylephrine for Paroxysmal Supraventricular Tachycardia during Lung Resection Surgery: A Case Report. The Korean Journal of Critical Care Medicine (KJCCM) 21(1): 57-62.

Kowalewski J, Brocki M, Dryjanski T, Kapron K, Barcikowski S. 1999. Right ventricular morphology and function after pulmonary resection. Eur J Cardiothorac Surg 15(4):444-8.

Kutlu CA, Williams EA, Evans TW, Pastorino U, Goldstraw P. 2000. Acute lung injury and acute respiratory distress syndrome after pulmonary resection. Ann Thorac Surg 69:376-80.

Lewis JW ,Bastanfar M, Gabriel F, Mascha E. 1994. Right heart function and prediction of respiratory morbidity in patients undergoing pneumonectomy with moderately severe cardiopulmonary dysfunction. J Thoracic Cardiovasc Surg 108(1):169-75.

Mageed NA, EL-Ghonaimy YA, Elgamal MA, Hamza U. 2005. Acute effects of lobectomy on right ventricular ejection fraction and mixed venous oxygen saturation. Ann Saudi Med 25:481-5.

Marik PE. 2013. Obituary: pulmonary artery catheter 1970 to 2013. Ann Intensive Care 3:38. 
McCall P, Corcoran D, Arthur A, et al. 2016. The right ventricular response to lung resection. J Cardiothorac Vasc Anesth 30:S23-4.

Okada M, Ota T ,Okada M ,Matsuda H ,Okada K, Ishii N. 1994. Right ventricular dysfunction after major pulmonary resection. J Thoracic Cardiovasc Surg 108(3):503-11.

Parquin F, Marchal M, Mehiri S, Hervé P, Lescot B. 1996. Post-pneumonectomy pulmonary edema: analysis and risk factors. Eur J Cardiothorac Surg 10(11):929-32.

Reed CE, Dorman BH, Spinale FG. 1996. Mechanisms of right ventricular dysfunction after pulmonary resection. Ann Thorac Surg 62(1):225-31.

Rena O, Papalia E, Oliaro A, et al. 2001. Supraventricular arrhythmias after resection surgery of the lung. Eur J Cardiothorac Surg 20:688-93.

Robin E, Costecalde M, Lebuffe G, Vallet B. 2006. Clinical relevance of data from the pulmonary artery catheter. Crit Care 10(Suppl 3):S3.

Rosenkranz S, Preston IR. 2015. Right heart catheterisation: best practice and pitfalls. Eur Respir Rev 24(138):642-52.

Sato Y, Nagata H, Inoda A, Miura H, Watanabe Y, Suzuki K. 2014. Cardioversion for paroxysmal supraventricular tachycardia during lung surgery in a patient with concealed Wolff-Parkinson-White syndrome. Masui 63:1106-10. [Article in Japanese]

Slinger P. 1999. Post-pneumonectomy pulmonary edema: Is anesthesia to blame? Curr Opin Anaesthesiol 12:49-54.
Tsuchida M, Yamato Y, Aoki T, et al. 2001. Complications associated with pulmonary resection in lung cancer patients on dialysis. Ann Thorac Surg 71:435-8.

Uno T ,Kitano T , Noguchi T ,Honda N , Chikama H, Tanaka K. 1993. The alteration of right ventricular performance in patients with pneumonectomy and pulmonary lobectomy. Masui 42(10):1459-63. [English MEDLINE] [Article in Japanese]

Vaporciyan AA, Correa AM, Rice DC, et al. 2004. Risk factors associated with atrial fibrillation after noncardiac thoracic surgery: analysis of 2588 patients. J Thorac Cardiovasc Surg 127:779-86.

Waller DA, Gebitekin C, Saunders NR, Walker DR. 1993. Noncardiogenic pulmonary edema complicating lung resection. Ann Thorac Surg 55(1):140-3

Wei B, D’Amico T, Samad Z, Hasan R, Berry MF. 2014. The impact of pulmonary hypertension on morbidity and mortality following major lung resection. Eur J Cardiothorac Surg 45:1028-33.

Wu DH, Xu MY, Mao T, Cao H, Wu DJ, Shen YF. 2012. Risk factors for intraoperative atrial fibrillation: a retrospective analysis of 10, 563 lung operations in a single center. Annals Thorac Surg 94:193-7.

Zeldin RA, Normandin D, Landtwing D, Peters RM. 1984. Postpneumonectomy pulmonary edema. J Thorac Cardiovasc Surg 87:359-65. [PubMed] 